\title{
HACIENDO-LUGAR EN TRÁNSITO. REFLEXIÓN SOBRE LA MIGRACIÓN AFRICANA Y TRABAJO DE CAMPO EN DARIÉN, PANAMÁ
}

\author{
Place-making in transit. Reflections on African migration \\ and fieldwork in Darién, Panama
}

Nanneke Winters*

\section{Introducción ${ }^{1}$}

Basado en trabajo de campo en la provincia de Darién, y desde la perspectiva de haciendo-lugar del dossier temático (véase Winters, Reiffen, 2019), esta reflexión pretende ofrecer una impresión de la migración de tránsito de personas migrantes africanas por Panamá. Ubicado como puente entre Sudamérica y Centroamérica, Panamá ha sido marcado como zona de paso por diferentes grupos de migrantes, incluso de africanos y africanas que viajan con el objetivo de llegar a Norteamérica. A pesar de la peligrosa selva de Darién, que en efecto sirve como una barrera natural entre Colombia y Panamá, en los últimos años el país ha visto una llegada llamativa de estas personas. La gran mayoría de ellas busca transitar el país lo más rápido posible, y como respuesta, el gobierno panameño últimamente ha aplicado una política de 'flujo controlado', en conjunto con su país vecino Costa Rica, para realizar este tránsito expedito. A través de reunir, revisar, vacunar, trasladar y transferir a las personas migrantes que salen de la selva, se mantiene un flujo tan controlado y muchas veces invisibilizado que se podría asumir que esta política no da espacio a que estas personas "hagan lugar".

Sin embargo, la presencia de personas migrantes africanas deja huellas en el territorio panameño. Siguiendo a estas huellas, en esta reflexión exploro tres dinámicas relacionadas: la angustia de una población; una estructura invisibilizada de acogida en comunidad indígena; y la posibilidad de

Instituto de Antropología y de Estudios Africanos de la Universidad Johannes Gutenberg de Mainz, Alemania. Mainz, Alemania. E-mail: nanneke.winters@uni-mainz.de. Orcid: 00000002-8036-3690.

1 Me gustaría agradecer al equipo del Servicio Jesuita para Migrantes Panamá por su colaboración amable y eficaz. También me gustaría agradecer a los y las participantes de investigación y revisores de esta reflexión. 
desplazamientos y emplazamientos permanentes. En conjunto, estas dinámicas iluminan cómo, incluso en zonas de paso, una perspectiva de haciendo-lugar podría ser útil para entender mejor los vínculos entre la visión gubernamental sobre ciertas migraciones, las prácticas de las personas migrantes; y el involucramiento de la población local.

El trabajo de campo sobre lo cual se reflexiona aquí se realizó en el marco del proyecto 'African trajectories across Central America. Dynamics of displacements, transitory emplacements, and ambivalent migration nodes' (Trayectorias africanas por Centroamérica. Dinámicas de desplazamientos, emplazamientos transitorios, y nodos de migración ambiguos) ${ }^{2}$. Este proyecto enfoca tanto en los desplazamientos y los viajes de las personas migrantes como en lo que nosotras llamamos los nodos de migración, con lo cual se refiere a varios sitios claves de vigilancia, coyotaje y solidaridad, en conjunto formando las trayectorias de las personas migrantes por los diferentes países centroamericanos. Hasta este momento, el proyecto incluye investigación etnográfica en Panamá y en Costa Rica. El trabajo de campo en Panamá duró un mes y medio en febrero-marzo 2019, y tomó lugar en la Ciudad de Panamá; la zona fronteriza norte de Paso Canoas; y la zona fronteriza sur de Darién. Esta reflexión enfoca en este último sitio, específicamente en el pueblo Metetí, compuesto por un casco urbano y varias comunidades indígenas. Trabajando en conjunto con el Servicio Jesuita para Migrantes, en Metetí conversé con varios actores claves de la migración africana, como agentes de la policía de fronteras (SENAFRONT); personal de varias ONGs; y misioneros y misioneras. También realicé observaciones en varios espacios claves de esta migración, como un albergue temporal y un centro de salud. Aunque, con excepción de la comunidad indígena que alberga a personas migrantes, había oportunidad para interactuar con la gente del pueblo, lamentablemente el acceso a las personas migrantes africanas era casi imposible dado la misma política del flujo controlado. Además, puesto que este trabajo de campo se realizó muy recientemente y es parte de un proyecto más amplio todavía por realizar, lo que ofrezco aquí es un primer acercamiento a dinámicas que nos pueden ayudar a entender mejor la situación migratoria actual en la zona.

\section{La angustia de una población}

Metetí, con aproximadamente 8,000 habitantes y localizado casi al final de donde la Interamericana se rompe por la selva de Darién, es un pueblo acostumbrado a la migración, o, mejor dicho, es un pueblo compuesto por migrantes. En los años 70, cuando el gobierno panameño decidió abrir esta

\footnotetext{
2 Proyecto en colaboración con la profesora Heike Drotbohm de la Universidad Johannes Gutenberg de Mainz, Alemania, financiado por Deutsche Forschungsgemeinschaft (DFG, 2018-2021, n. 406978565).
} 
sección de Darién, llegaron trabajadores afrocolombianos para construir la carretera y campesinos del interior del país, sobre todo de Chiriquí, para comprar terrenos a precios muy baratos, con el objetivo de deforestar la tierra para trabajarla y así expandir la frontera agrícola. Este proceso provocó desplazamientos en la población indígena, reforzando su larga historia de migraciones. Además, Metetí cuenta con la presencia de población asiática y sudamericana. Sin embargo, en los últimos años en Metetí han llegado personas migrantes no para quedarse, sino para pasar, ya sea por su propia cuenta y de forma irregular, o adentro del flujo controlado.

Aunque los habitantes insistan que el pueblo es solidario con las personas migrantes africanas que pasan, generalmente viéndolas como 'gente golpeada', también han llegado a tener miedo de asistirlas. Por su ubicación en región fronteriza, Metetí y su alrededor es una zona fuertemente controlada por SENAFRONT, que protege los diferentes intereses del Estado panameño. Se nota sus agentes en cada rincón del pueblo, ya sea en los puestos policiales en camino a y dentro de Metetí, en su puesto improvisado en el centro de salud, o en los restaurantes, tiendas e iglesias. Aunque la población de Metetí ya está acostumbrada a su presencia, reconociendo por ejemplo su trabajo de seguridad en el ámbito antidroga, agentes de SENAFRONT también son los y las que pueden arrestar a las personas sospechadas de facilitar la migración irregular. La esposa de un ex-taxista me contó, después de garantizarle la anonimidad, sobre la dura experiencia que su familia vivió cuando su esposo trasladó al hospital una migrante embarazada que encontró en el camino, para luego quedarse preso por dos semanas, acusado de tráfico de personas. Ella mencionó: "la policía no ve que eso deja a los hijos de uno desamparado. ... La ley no mira la parte humana", levantando su mano al corazón ${ }^{3}$. Según ella, la policía debería intentar a diferenciar mejor entre los diferentes casos de transportar y traficar a personas migrantes. Además, hace falta una docencia, en el sentido de que se lo explique a la población lo que conlleva el ofrecer ayuda. Porque, continúa: "es que nosotros somos campesinos, desde niños nos han inculcado estos valores, humilde, un plato de comida no se le niega a nadie" ${ }^{\prime 4}$. Aunque con la asistencia de un abogado su esposo salió libre, se quedaron con el susto, al igual que otras personas que lo han vivido de manera directa o indirecta.

En otro caso, un joven misionero se vio ante el dilema de acoger a personas migrantes que le tocaron la puerta después de días caminando en la selva. Al final él, con unos compañeros que estuvieron presentes en el momento, decidió darles reposo por una noche, pero con miedo. Él me contó:

\footnotetext{
3 Sin duda también hay personas que se aprovechan de esta situación y cobran a los migrantes de una manera excesivamente cara un servicio de transporte para evitar puestos de control.

4 Entrevista 6 marzo 2019.
} 
Les dijimos que lo que podemos hacer es hospedarlos esa noche, que se durmieran aquí, pero sí les pedimos pues que, por toda la misma situación que hay ahorita, ... que no le dijeran a nadie. ... Que no se comuniquen con nadie, que no digan el nombre del lugar porque, puede ser... Lo pueden perjudicar. Y ya luego ellos decidieron que sí, que iban a pasar la noche aquí, estaban muy cansados, comieron e inmediatamente se acostaron y, fueron a descansar porque los pobres estaban muy cansados. Y pues sí entre los tres se tomó la decisión de, de realmente corrernos el riesgo... En muchos sentidos.

Cuando le pregunté si podría explicar en qué sentido se corre riesgo, respondió:

Sí el riesgo es que con las autoridades que tenemos ahorita, si llegan a la casa de uno y ven migrantes ahí, ... las autoridades no van a decir que los migrantes vinieron a pedir ayuda. Las autoridades van a decir, no, ellos tienen escondido a los migrantes. Y es la problemática por lo cual ahorita mucha gente no es, o no se siente capaz, de apoyar al migrante. Porque, porque la gente tiene miedo, de que se vaya a tomar represalias con ellos, como ya es el caso de varias personas que han apoyado, que algunos incluso los han trasladado sin saber y sin cobrarles, los han trasladado en su carro de un punto a otro. Y ahora los tienen, por proceso de la ley tienen que firmar cada 15 días en un tribunal por, por solamente ayudar y claro, eso va limitando que la gente quiera ayudar. Porque, es lo que nosotros nos poníamos a pensar aquí, ¿̇y si vienen las autoridades, y los encuentran aquí, nosotros solamente les estamos dando de comer, les estamos dando donde duerman, pero... y si llegan [las autoridades] ${ }^{5}$

Aunque las personas migrantes que pasan no llegan a quedarse, su presencia parece generar una angustia al nivel del pueblo que cambia el tejido social de ello. Además, el tipo de ayuda que sí se puede brindar es sumamente controlada en estructuras formales, como veremos en la siguiente sección.

\section{Una estructura invisibilizada de acogida en comunidad indígena}

A lo largo de los últimos años, para responder a las personas migrantes que pasaban por el territorio alrededor del pueblo, las autoridades panameñas han intentado establecer varias estructuras para poder controlarles y brindarles ayuda en términos de agua, comida y salud. En Metetí se han utilizado, por ejemplo, un centro pastoral de la iglesia católica en el mero centro del pueblo, y un campamento temporal cerca de un hospital nuevo todavía por terminar. Por varias razones, entre ellas el descontento de una parte de la población local por la sobrecarga de estas estructuras, las mismas han sido abandonadas. Durante el trabajo de campo el gobierno panameño discutió planes de construir un albergue de más de ocho millones de dólares para acoger a migrantes, sin embargo, mucha gente no está de acuerdo con esta inversión (ver imagen 1). La

5 Entrevista 23 febrero 2019. 
sociedad civil local ha organizado varias reuniones para hablar del tema, ya que hay muchas necesidades en el pueblo, sobre todo en términos de agua potable y servicios de salud. Si localmente Darién es considerada como una provincia olvidada en términos de inversiones sociales por el Estado panameño, se podría esperar que no hay mucha disponibilidad para aceptar que se establezca una costosa estructura de acogida para personas migrantes que están de paso.

Imagen 1 - Dibujo publicado en el periódico popular El Siglo, 20 febrero 2019

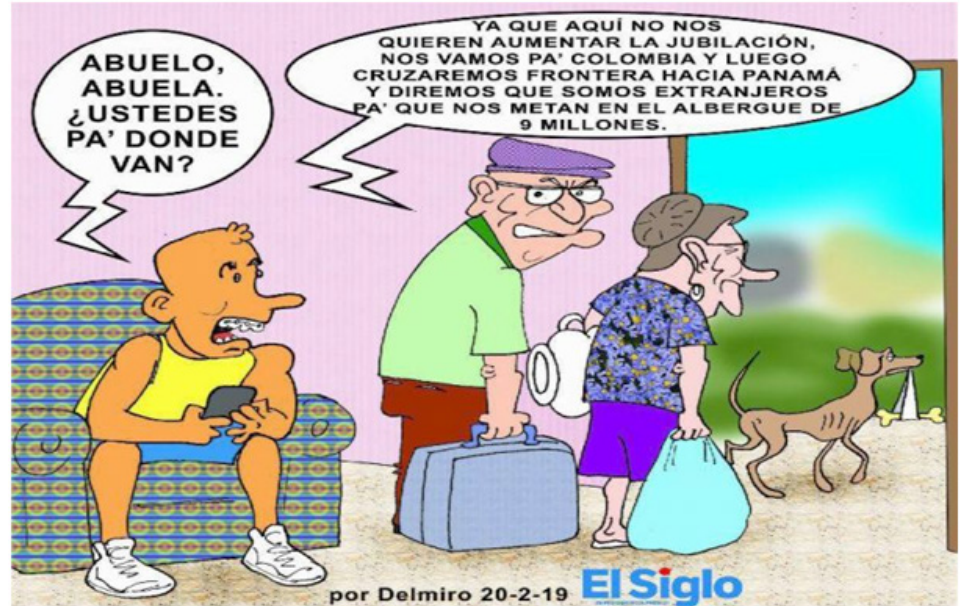

En este momento, para acoger a estas personas se está utilizando un 'albergue' en una comunidad que se llama La Peñita. Está ubicada a más o menos una hora de Metetí, primero siguiendo la Interamericana por media hora y después siguiendo un camino de difícil acceso en temporada de lluvia. En La Peñita llegan grupos de migrantes desde la selva y ahí las autoridades los mantienen hasta que Costa Rica los puede recibir y se les manda en bus hasta la frontera norte de Paso Canoas. Por mientras, las personas migrantes son contadas, revisadas y vacunadas. Las autoridades acumulan sus datos biométricos para compartirlos con Interpol y luego decidir sobre la continuación de su viaje. A menudo el albergue está sobrecargado por la llegada de nuevas personas migrantes. Durante el trabajo de campo la cantidad de migrantes que llegaba, acabó por superar al total de la población local. Por ejemplo, en nuestra primera visita a la comunidad habían casi 200 migrantes (entre ellos africanos, asiáticos, y haitianos), mientras según el agente responsable de SENAFRONT la población local cuenta unas 130 personas.

La Peñita es considerada como una comunidad indígena, y aunque durante nuestra estancia no logramos obtener acceso formal a esta comunidad ni al albergue establecido ahí, sí logramos obtener una primera impresión a 
través de nuestras visitas para pedir acceso y a través de las historias de otras personas trabajando en la zona. La impresión que obtuvimos es una de condiciones muy precarias, tanto para las personas migrantes como para la comunidad. Esta impresión urge a preguntar ìhasta qué grado las autoridades utilizan a la comunidad indígena para mantener a los migrantes lejos del centro del pueblo, afuera de vista, y qué consecuencias locales podría traer este proceso de aislamiento?

El albergue en La Peñita consiste de un hangar de zinc que anteriormente se utilizó para almacenar productos agrícolas. Adentro parece ser un solo espacio, sin divisiones, donde hace un calor tremendo. Afuera hay una pequeña área de cocina y hay algunos baños portátiles. El hangar está ubicado en medio de la comunidad, y alrededor, en sus casas, la población local ha hecho pequeños negocios para sustentar a las personas migrantes y generar un ingreso adicional, por ejemplo, vendiéndoles comida. En la entrada de La Peñita hay una lomita donde se asuman migrantes para intentar obtener señal de teléfono. Por la falta de agua potable, todo el mundo, incluso los agentes de SENAFRONT, se bañan en el río.

Este mismo río es el medio por lo cual muchas personas migrantes llegan a La Peñita, sea en lancha o siguiendo su curso a pie. Más arriba en la ruta que se utiliza para salir de la selva, está ubicada la comunidad indígena de Bajo Chiquito, donde SENAFRONT tiene presencia e intenta mantener a grupos de migrantes hasta que La Peñita se vacía y pueden seguir su camino. Sin embargo, Bajo Chiquito no cuenta con albergue. En cambio, las familias indígenas dan sus casas en alquiler a las personas migrantes, mientras ellas mismas a veces se retiran a sus fincas de donde sus niños no tienen acceso a la escuela. En temporadas de fuerte presencia de migrantes también se empeora la situación de escasez de agua, se reduce la capacidad para mantener una situación higiénica. Además, hay reportes de asalto a las personas migrantes en las Ilamadas trochas que llevan a Bajo Chiquito.

Parar a las personas migrantes en estas comunidades indígenas, revisarles y mantenerles hasta que se les pueda transportar a través del país, parece ser una manera útil para que la población central de Metetí no se dé cuenta de la cantidad de migrantes en la zona. Ya que aparte de agentes de SENAFRONT solamente es un grupo pequeño de trabajadores humanitarios/-as que llega a estas comunidades y se encuentra con migrantes, de manera errática y restringida. Su actual aislamiento podría ser una forma estratégica para evitar enfrentamientos entre pueblo y migrantes, para evitar descontento, y para mantener al pueblo tranquilo. Incluso, viendo el carácter específico de este aislamiento, iquizás se podría hablar de una re-marginalización de la comunidad indígena, que históricamente ya tiene que tratar con falta de recursos y derechos (véase Sánchez Saavedra, 2009)? Porque a pesar de que 
mucha gente de la comunidad indígena parece beneficiar de la presencia de las personas migrantes, a través de la venta de comida y de alquiler, entre otras cosas, no sabemos cuáles podrían ser los efectos más duraderos de esta situación. ¿De qué forma cambian estos negocios volátiles la economía de la comunidad? ¿Cuáles son las posibles consecuencias secundarias de la presencia de migrantes, por ejemplo, para la educación de los niños o para la disponibilidad del agua? Y, en términos generales, ¿̇en qué medida tiene la comunidad poder de decisión sobre la presencia del Estado y de las personas migrantes en su territorio?

\section{¿Desplazamientos y emplazamientos permanentes?}

Aunque las personas migrantes se han vueltas muy poco visibles en Metetí, la población local que es consciente de su presencia asuma que están de paso. Sin embargo, no todas logran pasar. Aparte de aquellas que son devueltas, la ruta por la selva es sumamente peligrosa y violenta y hay una cierta cantidad de personas que desaparecen en el camino. Por ejemplo, en la prensa panameña se ha comentado casos de ahogos en ríos selváticos (véase López Guía, 2016). En otros casos, agentes de SENAFRONT o miembros de las comunidades indígenas se encuentran con cuerpos o restos de cuerpos humanos en las trochas, víctimas de grupos armados, de animales silvestres, o de alguna enfermedad o algún accidente. Las autoridades intentan hacer la identificación y la autopsia, pero en muchos casos los cuerpos ya están tan dañados que los entierran ahí mismo donde los encontraron, en fosas comunes. En otros contextos, se ha abordado este tema del paisaje violento (véase De León, 2015) y se ha desarrollado proyectos de contar a personas migrantes que se han muertas (véase IOM, 2017). Como los datos sobre personas migrantes fallecidas en el Darién son muy escasos y de difícil acceso, me llamó la atención un caso de migrantes ghaneses que fueron enterrados/-as cerca de Metetí, en Santa Fe. Este caso me hizo reflexionar sobre las situaciones extremas de formar parte de y transformar un lugar.

Santa Fe es un pueblo con aproximadamente 7,000 habitantes a media hora de Metetí. Como está un poco más retirado de la Interamericana, normalmente no pasan migrantes ahí. Sin embargo, en el cementerio de Santa Fe hay una fosa de cinco migrantes ghaneses. Hace unos años, estas personas se ahogaron en un río cercano. Se les hizo la autopsia en la Ciudad de Panamá y, por alguna u otra razón que aún desconozco, les devolvieron a Santa Fe para ser enterradas ahí. Hasta donde se sepa, no se ha intentado o logrado establecer contactos institucionales para repatriarles a Ghana. Las autoridades les hicieron una fosa y les enterraron ahí según protocolo formal, en presencia de varias representantes del Estado y de la iglesia católica. Parece que pocas personas de Santa Fe se dieron cuenta de este entierro. Sin embargo, en el día 
de los muertos, algunas oraron por sus almas, como suelen hacer con personas sin parientes.

Algunos/-as miembros/-as de la sociedad civil luego investigaron lo sucedido y decidieron organizar un acto simbólico en el cementerio. En este acto, sembraron una semilla ghanesa en una llanta, marcando la fosa. Sin embargo, en el momento de trabajo de campo la fosa quedó sin marcar (ver imagen 2). Ya no hay rasgos visibles de la presencia de estas personas ghaneses, pero siguen enterradas ahí, en tierra ajena. La dificultad de encontrar a personas capaces de hablar del tema, y la dificultad de ubicar la fosa en sí, me llevó a reflexionar sobre lo sucedido casi como una historia borrada. Como un desplazamiento continuo a través de un emplazamiento literal y permanente.

Imagen 2 - Cementerio de Santa Fe. Al lado izquierdo de la llanta está ubicada la fosa no-marcada de los/-as migrantes ghaneses. Foto por la autora, 6 marzo 2019.

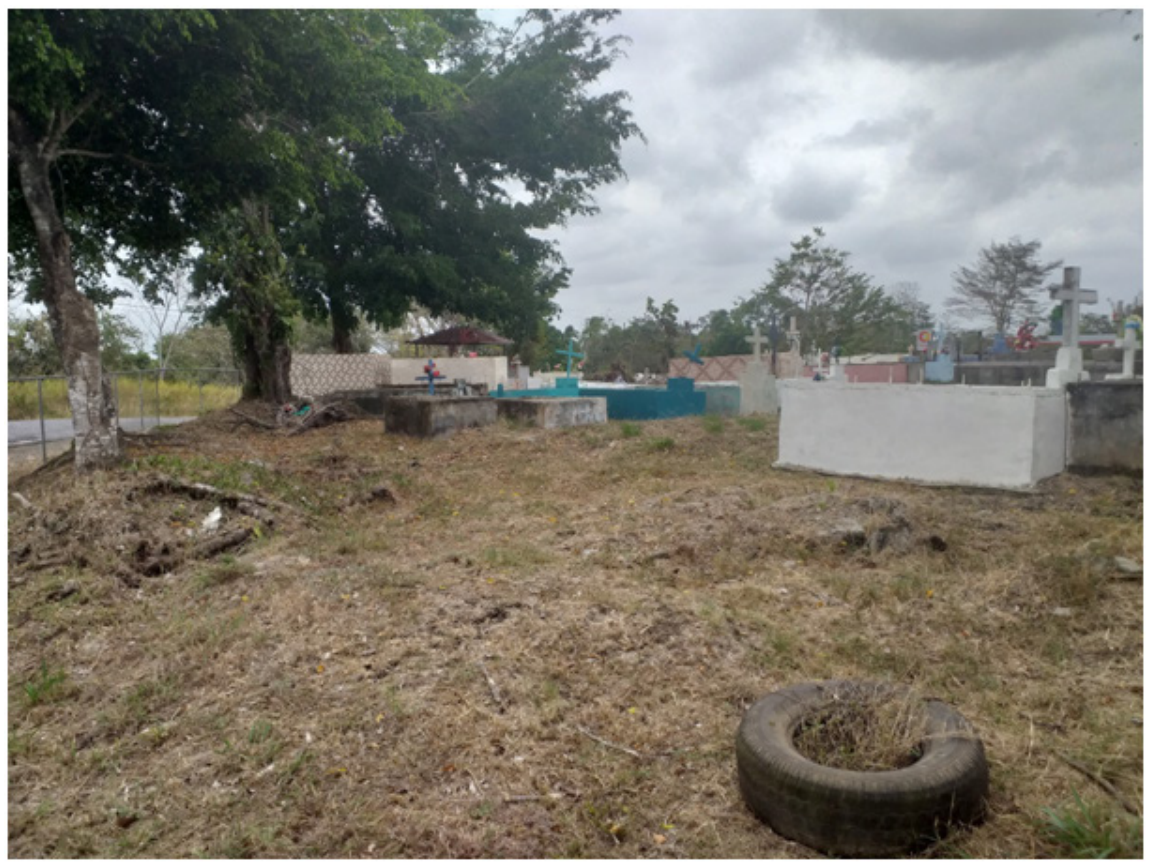

\section{Unas consideraciones finales}

En la introducción al dossier temático (Winters, Reiffen, 2019), Franziska Reiffen y yo distinguimos dos conjuntos de prácticas de haciendo-lugar. Por un lado, las prácticas de las personas migrantes que dejan huellas y por lo tanto se hacen parte del proceso de construir un lugar, y por otro, las prácticas que aportan al sentirse apegado e identificado con un lugar, de hacerlo suyo. 
Referimos a estos dos conjuntos de prácticas relacionados como haciendoLugar y Haciendo-lugar. En esta reflexión he intentado abordar el primer conjunto de prácticas a través de una exploración preliminar de tres dinámicas, basada en trabajo de campo en Panamá. Aunque estas prácticas ya iluminan un conjunto complejo de actores, sentimientos, decisiones políticas y actividades concretas involucrados en cómo las personas migrantes co-construyen un lugar, se podría pensar en una exploración más amplia.

En primer lugar, y siguiendo la terminología de la introducción, esta reflexión muestra la urgencia de pensar sobre haciendo-Lugar en espacios más allá de lo urbano y de lo céntrico, para llegar a una mejor comprensión de cómo diferentes lugares se ven involucrados en las trayectorias de las personas migrantes. Por ejemplo, se podrían incluir a comunidades indígenas como la de La Peñita y a sitios transfronterizos rurales. En segundo lugar, esta reflexión está limitada debido al difícil acceso a la población migrante africana en Darién, y, por lo tanto, no se sabe hasta qué nivel ella está involucrada en procesos de Haciendo-lugar. Viendo que, aún estando en tránsito, las personas migrantes pueden quedar varadas y de alguna u otra forma llegar a insertarse localmente, participando en y apropiando ciertos lugares (Drotbohm, Winters, 2018), hace falta una exploración más profunda de las posibilidades e imposibilidades de haciendo-lugar en situaciones de paso.

\section{Referencias}

DE LEÓN, Jason. The Land of Open Graves. Living and Dying on the Migrant Trail. Oakland: University of California Press, 2015.

DROTBOHM, Heike; WINTERS, Nanneke. Transnational Lives en Route: African Trajectories of Displacement and Emplacement across Central America. Working Papers of the Department of Anthropology and African Studies of the Johannes Gutenberg University Mainz, 175, 2018.

LÓPEZ GUÍA, Angel. Encuentran muerta a familia de migrantes en Darién; fallecieron ahogados en un río. La Prensa, 1 septiembre 2016. Disponible en: $<$ https://www.prensa.com/judiciales/Encuentran-migrantes-Darien-fallecieronahogados_0_4565543430.html >.

IOM. Fatal Journeys. Volume 3 PART 2. Improving Data on Missing Migrants. Geneva: International Organization for Migration, 2017.

SÁNCHEZ SAAVEDRA, Kevin E. Migración transfronteriza indígena. Los emberá y wounaan en Jaqué, Darién (tese de mestrado). Costa Rica: Ciudad Universitaria "Rodrigo Facio", 2009.

WINTERS, Nanneke; REIFFEN, Franziska. Haciendo-lugar vía huellas y apegos: las personas migrantes africanas y sus experiencias de movilidad, inmovilidad e inserción local en América Latina. Introducción al dossier temático REMHU 56. REMHU, Revista Interdisciplinar da Mobilidade Humana, v. 27, n. 56, ago. 2019, p. 11-33. 